\title{
The Explicit Inverses of Two Commonly Occurring Matrices
}

\author{
By D. Kershaw
}

Abstract. Explicit formulae are given for the inverses of certain tridiagonal scalar and block matrices.

During an investigation into the convergence properties of natural splines it was found convenient to have the explicit forms for the inverses of certain tridiagonal matrices. Special forms of these matrices arise in other branches of numerical analysis and so it may be useful to record them.

The derivation is elementary but complicated and will only be indicated.

\section{Notes.}

(i) The matrices will be of order $n \times n$.

(ii) $T_{r}, U_{r}$ will denote the Chebyshev polynomials of the first and second kinds respectively, both with argument $\lambda$.

(iii) The elements of $A, A^{-1}$ will be denoted by $a_{r s}, a_{r s}^{-1}$ respectively. Similarly for $B, B^{-1}$.

(iv) $U_{-1}=0, U_{-2}=-U_{0}$.

Matrix $A, n \geqq 2$.

$$
\begin{array}{rlrl}
a_{r s} & =-a & r=s=1, n, \\
& =-2 \lambda & r=s=2(1) n-1, & \\
& =1 \quad & |r-s|=1, \\
& =0 & & \text { otherwise }, \\
a_{r s}^{-1} & =\frac{-1}{a^{2} U_{n-2}-2 a U_{n-3}+U_{n-4}}\left[a U_{r-2}-U_{r-3}\right]\left[a U_{n-8-1}-U_{n-s-2}\right], \\
a_{s r}^{-1} & =a_{r s}^{-1} . & & 1 \leqq r \leqq s \leqq n,
\end{array}
$$

Matrix $B, n \geqq 3$.

$b_{r s}=a_{r s}, \quad$ except that $b_{1 n}=b_{n 1}=1$,

$b_{r s}^{-1}=\frac{-1}{a^{2} U_{n-2}-2 a U_{n-3}-2\left(1+T_{n-2}\right)}\left\{\begin{array}{c}{\left[a U_{r-2}-U_{r-3}\right]\left[a U_{n-8-1}-U_{n-8-2}\right]} \\ +U_{s-r-1}-U_{r-2} U_{n-8-1}\end{array}\right\}$,

$b_{s r}^{-1}=b_{r s}^{-1}$.

$1 \leqq r \leqq s \leqq n$.

\section{Special Forms of $A$.}

Received May 17, 1968. 


$$
\begin{aligned}
& a=2 \lambda \text {, }
\end{aligned}
$$

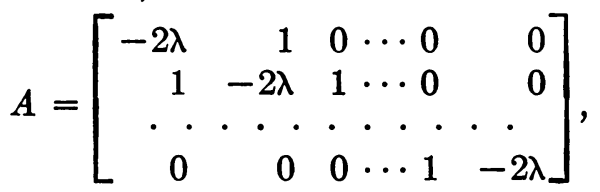

$$
\begin{aligned}
& a_{r s}^{-1}=-\frac{1}{U_{n}} \cdot U_{r-1} U_{n-s}, \quad 1 \leqq r \leqq s \leqq n, a_{s r}^{-1}=a_{r s}^{-1} \text {, } \\
& a=\lambda \text {, }
\end{aligned}
$$

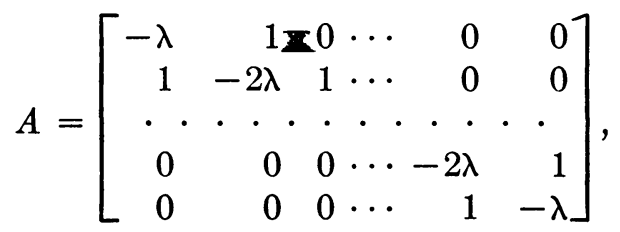

$$
\begin{aligned}
& a_{r s}^{-1}=\frac{1}{\left(1-\lambda^{2}\right) U_{n-2}} \cdot T_{r-1} T_{n-s}, \quad 1 \leqq r \leqq s \leqq n, a_{s r}^{-1}=a_{r s}^{-1} \text {. }
\end{aligned}
$$

\section{Special Forms of $B$.}

$$
\begin{aligned}
& a=2 \lambda \text {, }
\end{aligned}
$$

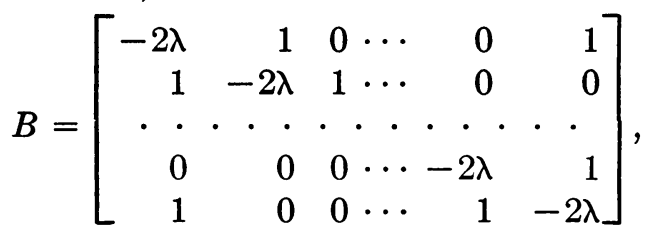

$$
\begin{aligned}
& b_{r s}^{-1}=\frac{1}{2\left[1-T_{n}\right]}\left[U_{n-s+r-1}+U_{s-r-1}\right], \quad 1 \leqq r_{i} \leqq\left[s \leqq n_{z}, b_{s r}^{-1}=b_{r s}^{-1},\right. \\
& a=\lambda \text {, }
\end{aligned}
$$

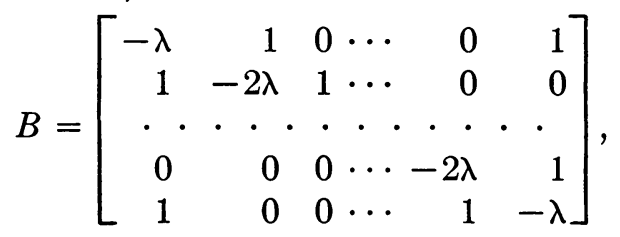

$$
\begin{aligned}
& b_{r s}^{-1}=\frac{1}{2+\left(2-\lambda^{2}\right) U_{n-2}}\left[T_{r-1} T_{n-s}-U_{r-2} U_{n-s-1}+U_{s-r-1}\right] \text {, } \\
& 1 \leqq r \leqq s \leqq n, b_{s r}^{-1}=b_{r s}^{-1} \text {. }
\end{aligned}
$$

Outlines of Proof for $A$. The columns of $A^{-1}$ are the solutions of

$$
A \mathrm{x}=\mathrm{e}_{\mathrm{s}}, \quad s=1,2, \cdots, n,
$$

where $\mathbf{e}_{\boldsymbol{s}}$ is the $s$ th unit vector. In recurrence form this matrix equation becomes

$$
\begin{aligned}
-a x_{1}+x_{2} & =0, \\
x_{r-1}-2 \lambda x_{r}+x_{r+1} & =\delta_{r s}, \quad r=1,2, \cdots, n-1, \\
x_{n-1}-a x_{n} & =0 .
\end{aligned}
$$


Note now that all except the first, last and sth equations are satisfied by either Chebyshev polynomial with argument $\lambda$. If one assumes, for example, that

$$
\begin{aligned}
& x_{r}=A T_{r}+B U_{r-1}, \quad r=1,2, \cdots, s-1, \\
& x_{r}=C T_{r}+D U_{r-1}, \quad r=s+1, \cdots, n,
\end{aligned}
$$

then each equation in (1) will be identically satisfied except for

$$
\begin{aligned}
-a x_{1}+x_{2} & =0, \\
x_{s-2}-2 \lambda x_{s-1}+x_{s} & =0, \\
x_{s-1}-2 \lambda x_{s}+x_{s+1} & =1, \\
x_{s}-2 \lambda x_{s+1}+x_{8+2} & =0, \\
x_{n-1}-a x_{n} & =0 .
\end{aligned}
$$

These give sufficient equations to solve for the unknowns $A, B, C, D, x_{s}$. A similar technique holds for $B$.

Generalization to Partitioned Matrices. If, in the matrices $A$ and $B$, the scalars $a, \lambda, 1$ are replaced by the $m \times m$ matrices $\Gamma, \Lambda, I$, ( $I$ being the unit matrix), respectively, then the results given above will still be valid if $\Gamma \Lambda=\Lambda \Gamma$, and the reciprocals which occur are replaced by the inverses of the corresponding matrices.

For example, the inverse of $A$, in block form, will be

$$
-\left[\Gamma^{2} U_{n-2}-2 \Gamma U_{n-3}+U_{n-4}\right]^{-1}\left[\Gamma U_{r-2}-U_{r-3}\right]\left[\Gamma U_{n-8-1}-U_{n-8-2}\right]
$$

where the argument of the Chebyshev polynomials is now $\Lambda$.

Department of Computer Science

University of Edinburgh

Edinburgh 8, Scotland 